\title{
Microbial community successional patterns in beach sands impacted by the Deepwater Horizon oil spill
}

\author{
Luis M Rodriguez-R ${ }^{1}$, Will A Overholt ${ }^{1}$, Christopher Hagan ${ }^{2}$, Markus Huettel², \\ Joel E Kostka ${ }^{1,3}$ and Konstantinos T Konstantinidis ${ }^{1,4}$ \\ ${ }^{1}$ School of Biology, Georgia Institute of Technology, Atlanta, GA, USA; ${ }^{2}$ Department of Earth, Ocean and \\ Atmospheric Sciences, Florida State University, Tallahassee, FL, USA; ${ }^{3}$ School of Earth and Atmospheric \\ Sciences, Georgia Institute of Technology, Atlanta, GA, USA and ${ }^{4}$ School of Civil and Environmental \\ Engineering, Georgia Institute of Technology, Atlanta, GA, USA
}

\begin{abstract}
Although petroleum hydrocarbons discharged from the Deepwater Horizon (DWH) blowout were shown to have a pronounced impact on indigenous microbial communities in the Gulf of Mexico, effects on nearshore or coastal ecosystems remain understudied. This study investigated the successional patterns of functional and taxonomic diversity for over 1 year after the DWH oil was deposited on Pensacola Beach sands (FL, USA), using metagenomic and 16S rRNA gene amplicon techniques. Gamma- and Alphaproteobacteria were enriched in oiled sediments, in corroboration of previous studies. In contrast to previous studies, we observed an increase in the functional diversity of the community in response to oil contamination and a functional transition from generalist populations within 4 months after oil came ashore to specialists a year later, when oil was undetectable. At the latter time point, a typical beach community had reestablished that showed little to no evidence of oil hydrocarbon degradation potential, was enriched in archaeal taxa known to be sensitive to xenobiotics, but differed significantly from the community before the oil spill. Further, a clear succession pattern was observed, where early responders to oil contamination, likely degrading aliphatic hydrocarbons, were replaced after 3 months by populations capable of aromatic hydrocarbon decomposition. Collectively, our results advance the understanding of how natural benthic microbial communities respond to crude oil perturbation, supporting the specializationdisturbance hypothesis; that is, the expectation that disturbance favors generalists, while providing (microbial) indicator species and genes for the chemical evolution of oil hydrocarbons during degradation and weathering.
\end{abstract}

The ISME Journal (2015) 9, 1928-1940; doi:10.1038/ismej.2015.5; published online 17 February 2015

\section{Introduction}

The oil spill caused by the blowout of the Deepwater Horizon (DWH). Drilling rig in April 2010 constitutes the largest accidental release of oil into the marine environment in recorded history. Oil contamination from the DWH spill had a profound impact on indigenous microbial communities, and all available studies recognize shifts in the composition of microbial communities in direct contact with oiled seawater and sediments in comparison with pristine environments (Atlas and Hazen, 2011; Joye et al., 2014; Kostka et al., 2014; King et al., 2015). Moreover, consistent patterns were observed in microbial communities exposed to DWH oil in the

Correspondence: KT Konstantinidis, Civil and Environmental Engineering, Georgia Institute of Technology, 311 Ferst Dr, Ford ES\&T Building, Suite 3224, Atlanta, GA 30332, USA.

E-mail: kostas@ce.gatech.edu

Received 12 September 2014; revised 16 December 2014; accepted 23 December 2014; published online 17 February 2015
Gulf of Mexico including an increase in the relative abundance of members of the Gammaproteobacteria, a prevalence of known hydrocarbon-degrading populations, and the enriched abundance and expression of genes related to hydrocarbon degradation (Joye et al., 2014; Kostka et al., 2014; King et al., 2015). These patterns and microbial responses are also in accordance with observations from laboratory studies and previous accidental releases of oil in marine environments (Röling et al., 2002; Head et al., 2006; Yakimov et al., 2007; Berthe-Corti and Nachtkamp, 2010; Greer, 2010; McGenity et al., 2012).

The Unified Area Command estimated that approximately one-half of the $\sim 4.9$ million barrels of oil released from the DWH blowout reached the ocean surface (Lubchenco et al., 2010), and a portion of this surfaced oil transported to nearshore and coastal ecosystems was buried in the sediments (Hayworth et al., 2011; Wang and Roberts, 2013), impacting approximately $850 \mathrm{~km}$ of beaches from east Texas to west Florida (Michel et al., 2013). Oil started depositing on the Pensacola Beach sands 
studied here on 22 June 2010. The input of large amounts of crude oil, including an array of potentially toxic compounds, posed a potential disturbance for benthic microbial communities (Valentine et al., 2012). Available studies to date were primarily focused on the water column and/or deep sea ecosystems, and less is known about the response or adaptation of sedimentary communities to oiling (Huettel et al., 2014). Studies characterizing the taxonomic shifts between contaminated and noncontaminated beach sediments recognized that the oil input strongly affected the beach sand microbial communities, which responded with increased bacterial cell densities (Kostka et al., 2011), reduced taxonomic diversity, and a succession of microbial populations that paralleled the changes in abundance and composition of deposited hydrocarbons (Kostka et al., 2011; Bik et al., 2012; Lamendella et al., 2014). Consistent responses have been observed across study sites, although other factors such as site heterogeneity and seasonal fluctuations in environmental parameters have been shown to somewhat confound assessments of the oil impact in certain beaches (Newton et al., 2013), sometimes making them undetectable (Röling et al., 2004). In general, an initial increase in the relative representation of known oil degraders, mostly of the Gammaproteobacteria class (most notably Alcanivorax), was observed together with a temporal succession characterized by an increase in relative abundance of Bacillus, Microbacterium and members of the Alphaproteobacteria class at later stages, when recalcitrant oil hydrocarbons predominate (Kostka et al., 2011). Moreover, the increase in oil degraders was concomitant with an increased expression of polycyclic aromatic hydrocarbons, $n$-alkane and toluene degradation genes as assessed by metatranscriptomics (Lamendella et al., 2014). Although these findings provided important insights into the effects of oil on benthic microbial community composition, the gene functions selected for and the genomic adaptations in response to the presence of oil remained mostly uncharacterized in the Gulf coast.

Previously identified shifts in microbial communities in response to DWH oil, both in the water column and sediments, indicated significant susceptibility of these communities; susceptibility defined as the degree to which community composition changes in response to disturbance (Shade et al., 2012). These observations are in accordance with the majority of ecological studies addressing the effect of disturbances such as carbon inputs on microbial communities, which have found evidence of susceptibility (reviewed by Allison and Martiny, 2008). However, the magnitude, stability and stochasticity of functional responses, as well as the mechanisms driving the taxonomic and functional composition of the microbial community after disturbance are not well understood (Reed and Martiny, 2007). For example, it has been recognized in plant and animal communities that generalist populations better withstand disturbances, whereas specialist populations tend to be favored in stable environments (specialization-disturbance hypothesis; Vázquez and Simberloff, 2002). According to the disturbance-specialization hypothesis, most specialist taxa are selected against when communities experience a severe disturbance, as they are adapted to relatively narrow niches in their natural ecosystem. In contrast, generalists are more resilient to disturbances altering the niches. In turn, the taxonomic diversity of the community is negatively impacted by a disturbance, but the functional diversity can increase as an effect of the disturbance. Although some previous studies applied ecological theory to describe the response and recovery of community dynamics to disturbance ( $c f$. Prosser et al., 2007; Shade et al., 2012), the relationship of disturbance and specialization remains largely unexplored in microbial communities. Disturbed communities are typically observed to encompass reduced taxonomic and/or phylogenetic diversity compared with undisturbed controls, but whether this pattern translates to reduced functional diversity or increased specialization remains largely unknown. In this study, we aimed to characterize the response of sedimentary microbial communities from Pensacola Beach to the DWH oil spill, as an in-situ experiment of the effects of disturbance on functional and taxonomic diversity.

\section{Materials and methods}

Beach sands were collected at Pensacola Municipal Beach, FL, USA $\left(30^{\circ} 19.57 \mathrm{~N}, 087^{\circ} 10.47 \mathrm{~W}\right)$ on 6,10 , 20 and 24 May 2010 (before arrival of the oil plume to the shoreline; hereafter, termed pre-oil communities/samples), 30 July 2010 (one month after the oil reached the beach; oiled), 20 October 2010 (when oil constituents were still present in the sand; weathered oiled), and 14 June 2011 (when oil was not visually detectable; recovered; Table 1 and Supporting methods). Samples were collected from aerobic beach sediments (oxygen concentrations between 210 and $230 \mu \mathrm{moll}^{-1}$ down to $55 \mathrm{~cm}$ depth, which represents $>50 \%$ of air saturation level) above groundwater level.

16S rRNA gene amplicons were sequenced, and the resulting sequences were analyzed as described recently (Poretsky et al., 2014). Trimmed sequences were clustered into operational taxonomic units (OTUs) at 97\% similarity using UCLUST (Edgar, 2010 ), OTUs that represented $<0.005 \%$ of the total sequences were discarded (Bokulich et al., 2013) and representative sequences of each OTU were classified using the RDP Classifier at 50\% confidence (Wang et al., 2007). Shotgun community DNA was sequenced, and the resulting metagenomic reads were quality checked, assembled and annotated as described in the supplementary online 
Table 1 Samples used in this study

\begin{tabular}{|c|c|c|c|c|c|}
\hline Designation & Reads after trimming ${ }^{a}$ & Status $^{b}$ & Depth (cm) & Sampling date & Sediment temp. $\left({ }^{\circ} \mathrm{C}\right)^{\circ}$ \\
\hline S1 & 2937972 & Pre-oil & 0 & 6 May 2010 & $29.96 \pm 2.66^{\mathrm{d}}$ \\
\hline S2 & 7951456 & Pre-oil & 0 & 10 May 2010 & \\
\hline S3 & 7837390 & Pre-oil & 0 & 20 May 2010 & \\
\hline S4 & 6710972 & Pre-oil & 0 & 24 May 2010 & \\
\hline A & 32840836 & Oiled & $30-40$ & $30 \mathrm{Jul} 2010$ & $30.49 \pm 2.72$ \\
\hline B & 32392430 & Oiled & 35 & & \\
\hline $\mathrm{C}$ & 25024134 & Oiled & $30-40$ & & \\
\hline D & 21469632 & Weathered oil & $48-62$ & & \\
\hline $\mathrm{E}$ & 26279070 & Oiled & $40-45$ & 20 Oct 2010 & $23.73 \pm 2.95$ \\
\hline $\mathrm{F}$ & 34830190 & Oiled & $25-47$ & & \\
\hline G & 39208672 & Oiled & $24-36$ & & \\
\hline $\mathrm{H}$ & 25224316 & Weathered oil & $50-55$ & & \\
\hline I600 & 33188686 & Recovered & $30-40$ & 14 Jun 2011 & $31.02 \pm 2.79$ \\
\hline I606 & 31477910 & Recovered & $30-40$ & & \\
\hline J598 & 31724116 & Recovered & $50-65$ & & \\
\hline J604 & 28119496 & Recovered & $50-65$ & & \\
\hline
\end{tabular}

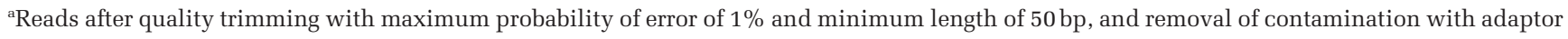
sequences.

bamples with oiled and weathered oil status were distinguished based on visual assessment of oil presence. Recovered status was defined based on undetectable levels of hydrocarbons at depths similar to (previously) oiled samples.

'Sediment temperature between 0 and $50 \mathrm{~cm}$ depth presented as mean \pm one standard deviation.

${ }^{\mathrm{d}}$ Data for May 2010 not available, presented values were measured in June 2010. Cf. temperatures in May 2011: 25.21 \pm 2.07.

material. The level of coverage of the community achieved by each metagenomic dataset was estimated and projected using Nonpareil with default parameters (Rodriguez- $\mathrm{R}$ and Konstantinidis, 2014b). Assembled contigs were taxonomically annotated using MyTaxa (Luo et al., 2014). 18S rRNA gene-encoding reads were identified by Metaxa (Bengtsson et al., 2011) with e-value $<0.1$ and taxonomically annotated using pplacer and taxtastic (Matsen et al., 2010). Read mapping to estimate the relative abundance of genes and taxa was performed using BLAT with default parameters (Kent, 2002), considering only the best match with alignment length $\geqslant 80 \mathrm{bp}$ and identity $\geqslant 97 \%$. Annotation terms and taxa with significantly different abundance between groups of samples were identified using the negative binomial test as implemented in DESeq2 (Anders and Huber, 2010).

To measure the average number of genes per cell with a given functional annotation (genome equivalents), a set of universally conserved single-copy genes were identified among the assembled gene sequences from the metagenomes. All genes were compared against a collection of 101 HMMs (Dupont et al., 2012), using HMMER3 (http://hmmer.janelia.org/) with default settings and trusted cutoff, excluding genes for which more than one model represented the same gene family. The median sequencing depth (in reads/bp) of the remaining 91 models was used as the normalizing factor for each dataset. The sequencing depth of genes with a given annotation (see below) was estimated for each dataset (in reads/bp), added up and divided by the normalizing factor of the corresponding dataset.

To identify genes related to oil degradation, gene-specific databases were compiled and manually curated. Sequences for AlkB (alkane hydroxylase) and CYP153 (cytochrome P450 family) were derived from the annotated datasets by Wang et al. (2010); sequences for NahA (naphthalene 1,2-dioxygenase) were derived from the set compiled by Lu et al., 2012; and sequences for ArhA (polycyclic aromatic hydrocarbon dioxygenase) and BBS (benzylsuccinyl-CoA dehydrogenase) were derived from UniRef50 clusters (Suzek et al., 2007). Putative proteins of the assembled metagenomes were functionally identified using blastp (Altschul et al., 1990) against each reference dataset, with a 250 bit-score threshold. The resulting dataset for AlkB was aligned using Muscle v3.8.31 with default parameters (Edgar, 2004), and the gene phylogeny was reconstructed using RAxML v7.7.2 with GTR model (proteins), gamma parameter optimization, and '-f a' algorithm (Stamatakis, 2006). Putative coding fragments predicted with FragGeneScan (Rho et al., 2010) on sequence reads were subsequently placed onto the reconstructed tree based on a sequence-to-profile alignment built with Clustal Omega v1.1.0 (Sievers et al., 2011), using the evolutionary placement algorithm (Berger et al., 2011). The same placement strategy was independently applied to the partial sequences of AlkB reported in the study by Smith et al. (2013) (GenBank entries KF613175-KF613575).

Diversity was calculated as the true diversity of order one $\left({ }^{1} D\right.$; equivalent to the exponential of Shannon index). The $\alpha$ and $\gamma$ components were estimated from the abundance of categories in a sample and in all samples, respectively, and adjusted for unobserved fractions using the ChaoShen correction (Chao and Shen, 2003) as implemented in the $\mathrm{R}$ package entropy (Hausser and Strimer, 2013). Richness was estimated using the Chao1 index (Chao, 1984), and evenness was calculated as the corrected true diversity of order 
one (number of equivalent groups) divided by the estimated richness (number of groups).

Crude oil hydrocarbons in the sediment samples were identified by gas chromatography-mass spectrometry using an Agilent 7890A Series GC (Santa Clara, CA, USA), coupled to an Agilent 7000 triple quadrupole MS system, as described previously (Zuijdgeest and Huettel, 2012). The supplementary online material provides further information about procedures and analytical techniques.

All sequencing datasets were deposited in the NCBI Sequence Read Archive under project PRJNA260285 and additional material is available at http://enve-omics.ce.gatech.edu/data/oilspill.

\section{Results}

Description of samples and their metagenomes Concentrations of total petroleum hydrocarbons quantified by gas chromatography-mass spectrometry and visible oil stains monotonically decreased between sampling dates ( $P$-values $\leqslant 0.05$, one-sided $t$-test; Figure 1a). Specifically, the depth-integrated sedimentary inventories of small molecular weight aliphatic and aromatic compounds decreased rapidly from 6 and $1 \mathrm{mg} \mathrm{kg}^{-1}$, respectively, in July to less than $0.5 \mathrm{mg} \mathrm{kg}^{-1}$ in October. In contrast, gas chromatography-mass spectrometry profiles revealed that sedimentary inventories of aromatic compounds greater than C8 remained unchanged during this same time frame, whereas aliphatic compounds greater than C6 displayed only a marginal reduction.

A total of 16 metagenomic samples, ranging in size from 3 to 78 million reads after trimming (paired-end reads with average length of 90-190 bp per dataset), were recovered from each of the four sampling time points, with at least three replicates per time point (Table 1). The metagenomes from preoil samples had an estimated abundance-weighted average coverage (Rodriguez- $R$ and Konstantinidis, $2014 \mathrm{~b}$ ) of $18-39 \%$, the oiled samples a coverage of $35-60 \%$ and the samples from recovered communities an average coverage of $20-25 \%$. Nonpareil curves indicated that the communities in the recovered samples had a higher sequence complexity than both pre-oil and oiled communities, with pre-oiled communities displaying a slightly lower sequence complexity (Supplementary Figure S1A). The described trend in sequence complexity corresponded to the estimated richness of these communities based on OTUs from 16S rRNA gene amplicon data (Supplementary Figure S1B). In general, all metagenomes showed lower sequence complexity than previously determined metagenomes from
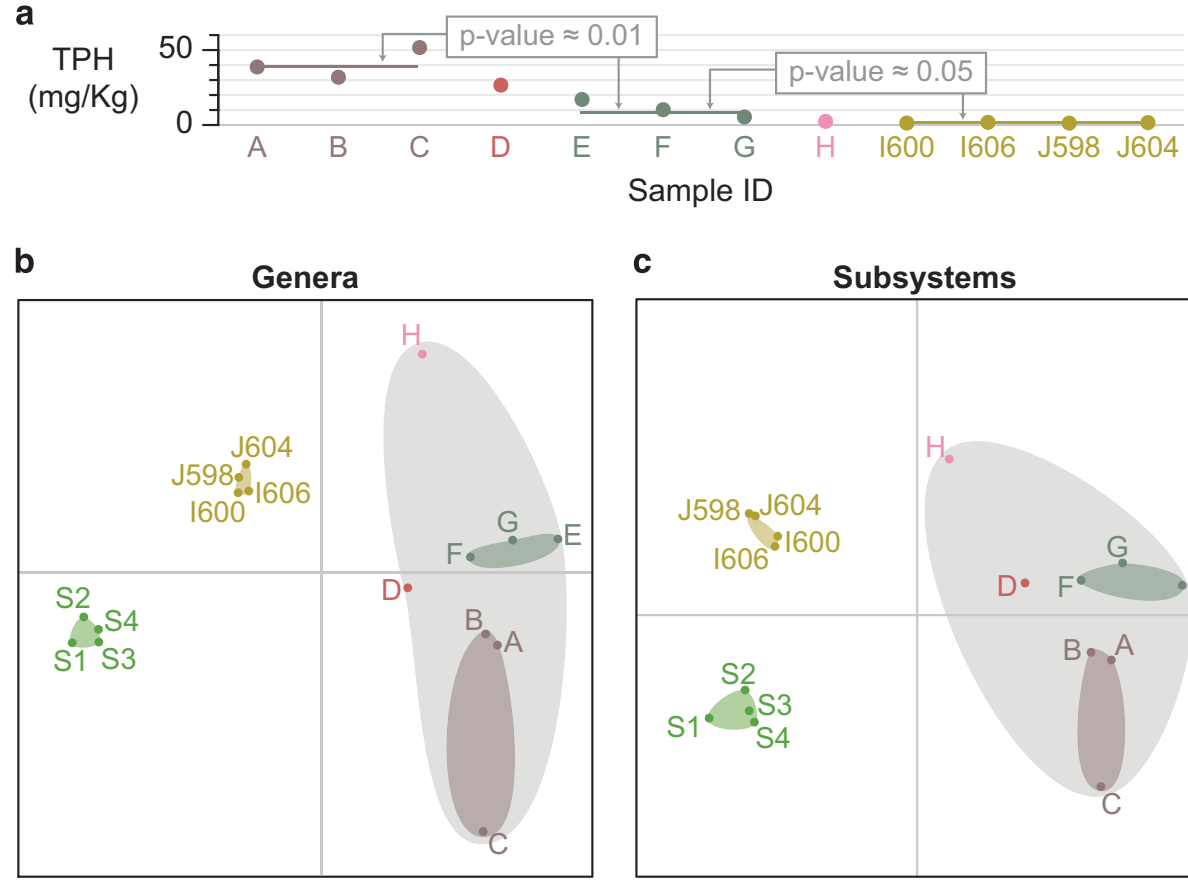

c

Subsystems

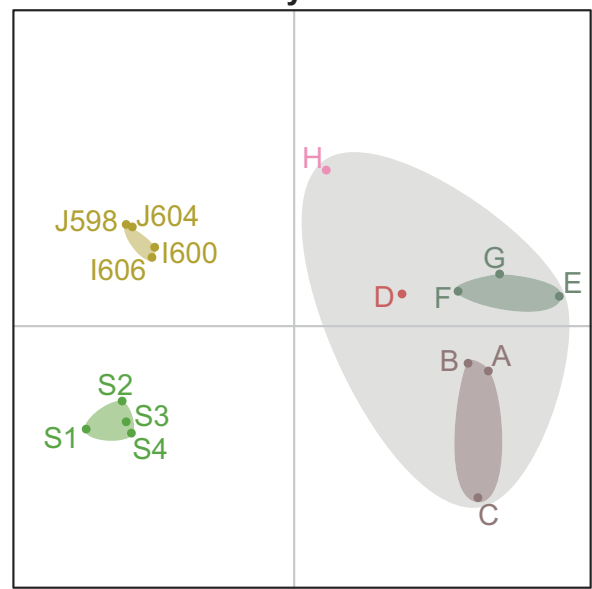

Figure 1 Shifts in taxonomic and functional profiles in relation to oil concentration. (a) The concentration of total petroleum hydrocarbons was significantly higher in July samples (A, B, C) relative to October 2010 (E, F, G), and June 2011 samples (I600, I606, J598, J604). The comparisons between groups (July to October 2010, and October 2010 to June 2011) were performed using one-sided $t$-tests ( $P$-values in grey boxes), and the average per group is indicated as horizontal lines. The non-metric multidimensional scaling of (b) genera and (c) subsystems reveals non-overlapping regions between pre-oiled (green), oiled (shadowed grey) and recovered (olive) samples. The two-dimensional stresses for genera and subsystems are $3.361 \%$ and $3.358 \%$, respectively, and the origins are indicated with grey lines. Distance matrices were generated using Bray-Curtis dissimilarities of normalized read counts and ordination was selected by minimizing stress on two dimensions. Note that the heavily oiled samples also form non-overlapping areas by sampling date (dark green and brown), and are distinguishable from weathered oil samples (pink and dark red). 
clayey or silty soils such as rain forest and permafrost but were more complex than freshwater or ocean planktonic metagenomes (Supplementary Figure S1A; $c f$. Rodriguez-R and Konstantinidis, 2014a). The July and October 2010 samples (oiled and weathered oil) were assembled into $\sim 56000$ contigs per sample with N50 of $\sim 1400 \mathrm{bp}$; while those from recovered samples resulted in $\sim 12000$ contigs per sample with N50 of $\sim 745 \mathrm{bp}$ (Supplementary File S1). These results further supported the Nonpareil estimates of higher sequence complexity in the latter samples. In total, $\sim 670000$ contigs were obtained with an overall N50 of $1101 \mathrm{bp}$ (723 Mbp in total, from $37 \mathrm{Gbp}$ of sequencing reads), on which $\sim 1.2$ million genes were predicted, resulting in an average coding density of $87 \%$ (Supplementary File S1).

\section{Microbial community specialization in response to oiling}

To assess the temporal effects of the oil spill on the microbial community composition and its recovery, the functional and taxonomic profiles at different time points were compared. Four main groups were identified which significantly differed in both taxonomic and functional distributions ( $P$-values $\leqslant 0.003$, ANOSIM based on Bray-Curtis dissimilarity; Figures $1 \mathrm{~b}$ and $\mathrm{c}$ ) and were consistent with the oil concentrations measured in-situ: Pre-oil (S1, S2, S3, and S4), Oiled July 2010 (A, B, C), Oiled October 2010 (E, F, G) and Recovered (I600, I606, J598, J604). $16 S$ rRNA gene amplicon data also demonstrated that sample depth played a limited role in structuring microbial communities (Supplementary Figure S2; ADONIS: $3 \%$ variance explained by depth vs $75 \%$ explained by oiling status and collection date), which was consistent with the facts that the beach sands studied here are subjected to high levels of erosion, and high levels of oxygen ( $>50 \%$ of air saturation level) were detectable at all sampling depths. Hence, our pre-oiled datasets, even though originated from different depths (surficial) compared to oiled datasets $(30-65 \mathrm{~cm})$, represented reliable controls for assessing the oiled and recovered microbial communities.

Most notably, the communities exhibited an increase in the functional diversity in oiled samples with respect to pre-oil samples, and a reduction in functional diversity in recovered samples with respect to oiled samples (Supplementary Figure S3A), revealing a different state of lower functional diversity in the recovered communities (Supplementary Figure S3B; DECORANA analysis). Interestingly, this pattern was not observed in the taxonomic diversity, richness or evenness levels (Supplementary Figure S3C-E), indicating that it was primarily due to a decrease in functional specialization of the communities in the oiled samples. This interpretation is further supported by a concomitant decrease in the estimated minimum doubling time in the oiled communities (Supplementary Figure S4A), as expected for bacteria with more generalist strategies (Dethlefsen and Schmidt, 2007). More generalist prokaryotes tend to have larger genomes (Konstantinidis and Tiedje, 2005), but no significant changes in the estimated average genome size were detected (Supplementary Figure S4B). Nevertheless, these results suggested that the oil disturbance caused community shifts characterized by a decrease in functional specialization and a consequent increase in functional diversity, which were reversed in the post-disturbance recovery process as the succession advanced.

\section{Oil degradation and toxicity drives community \\ phylogenetic composition}

Differences in the composition of the communities from pre-oil, oiled and recovered sediments were detected at various levels of taxonomic resolution (Figure 2; Supplementary File S3). At the most general level (domain), recovered communities exhibited higher fractions of eukaryotic and archaeal members than oiled and pre-oiled communities (Figure 3a), although no differences in the taxonomic composition of the eukaryotic fraction were observed (Supplementary File S3). The higher fraction of eukaryotic sequences is also consistent with the lower coding potential of May, July and October 2010 metagenomes $(\sim 89 \%$ of total sequence length was protein-coding) vs the Recovered (June 2011) metagenomes (70\%; Supplementary File S1). The higher representation of dominant taxa and lower evenness in communities from oiled samples was also evident at the class level, where Gamma- and Alphaproteobacteria increased in abundance, with a concomitant decrease of novel taxa (represented by the unclassified fraction; Figure 2b). The genera significantly more abundant in oiled than in pre-oiled and/or recovered samples were primarily well-known and suspected hydrocarbon degraders, including Alcanivorax, Pseudomonas, Hyphomonas, Parvibaculum, Marinobacter and Micavibrio (Figure 2c). In contrast, groups significantly enriched in recovered samples included taxa typically found in marine environments and known to be highly susceptible to xenobiotics such as the archaeal genera Nitrosopumilus and Cenarchaeum.

\section{Functional gene content shift in response to oil}

To further investigate the specific functional traits selected by oil presence and, presumably, accounted for the community compositional shifts observed, the abundances of genes associated with alkane and aromatic degradation pathways were compared between pre-oil, oiled and recovered samples. In all evaluated cases, oiled communities displayed a larger prevalence of gene annotations associated with aromatic and alkane hydrocarbon degradation 
a

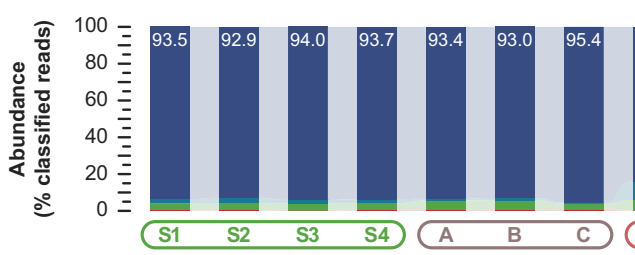

Domain: Bacteria $\square$ Archaea $\square$ Eucarya $\square$ Vira

b

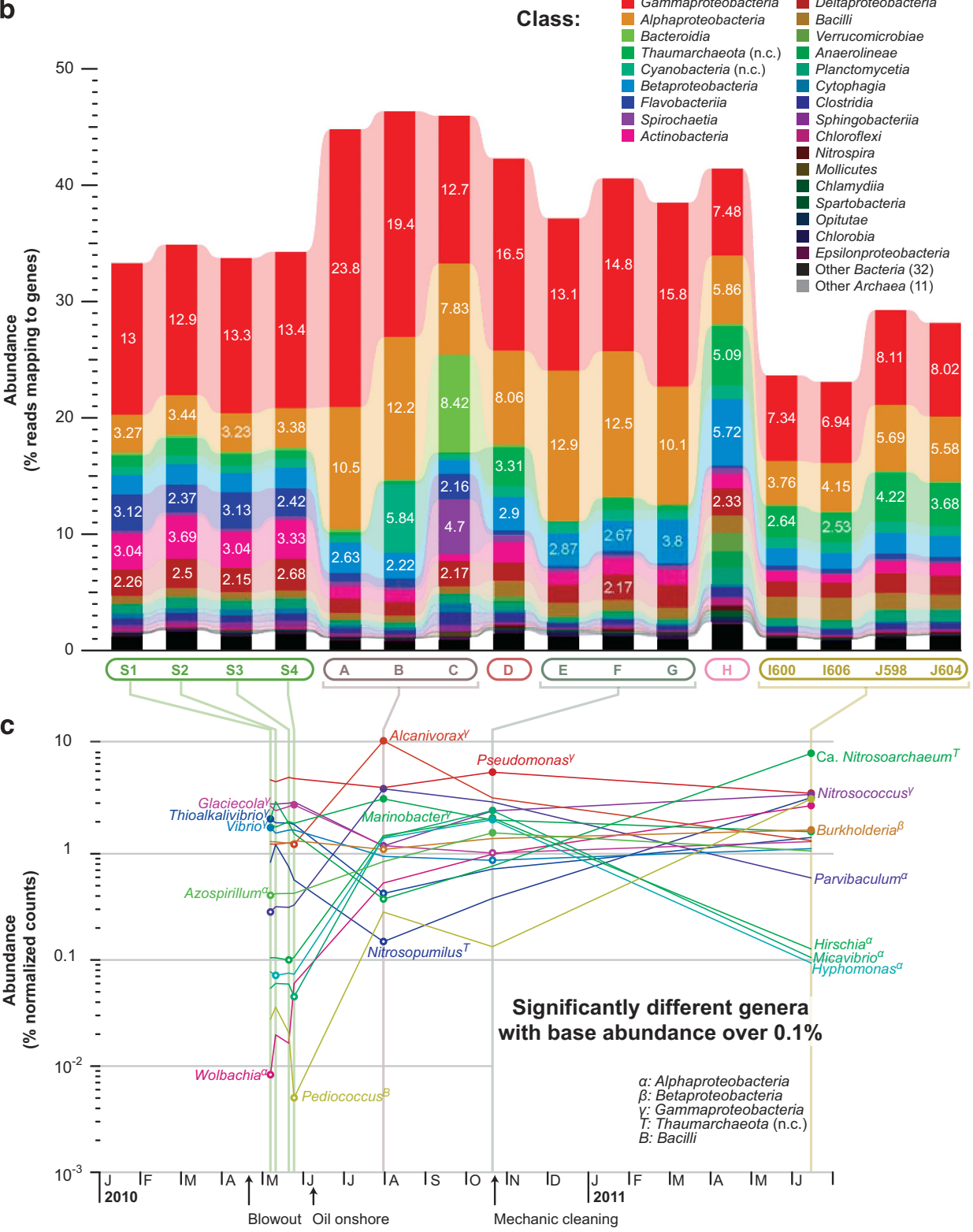

Figure 2 Taxonomic shifts in the microbial community in response to oil. The distribution of metagenomic reads in (a) domains and (b) classes is displayed for taxa that recruited more than $10 \%$ and $2 \%$ of the total reads, respectively (white numbers). (c) Genera with abundance above $0.1 \%$ and significantly different between pre-spill and oiled or between oiled and recovered samples $(P$-value adjusted $\leqslant 0.01)$ are also displayed. The minimum and maximum abundance of each genus is indicated with open and filled circles, respectively, and the class is indicated with superscripts.

as well as beta-oxidation than pre-oil and recovered communities (Figure 3). Interestingly, the relative abundance of most genes associated with aliphatics degradation dropped from July to October 2010, in particular those associated with rubredoxin-NAD + / NADP reduction and aldehyde oxidation (top panel in Figure 3). In contrast, the abundance of genes associated with aromatics degradation was roughly maintained or, in some cases, increased from July to October 2010 (second panel in Figure 3). In addition, functions related to nutrient scavenging such as allantoicase and nitrogenase (low nitrogen response), 


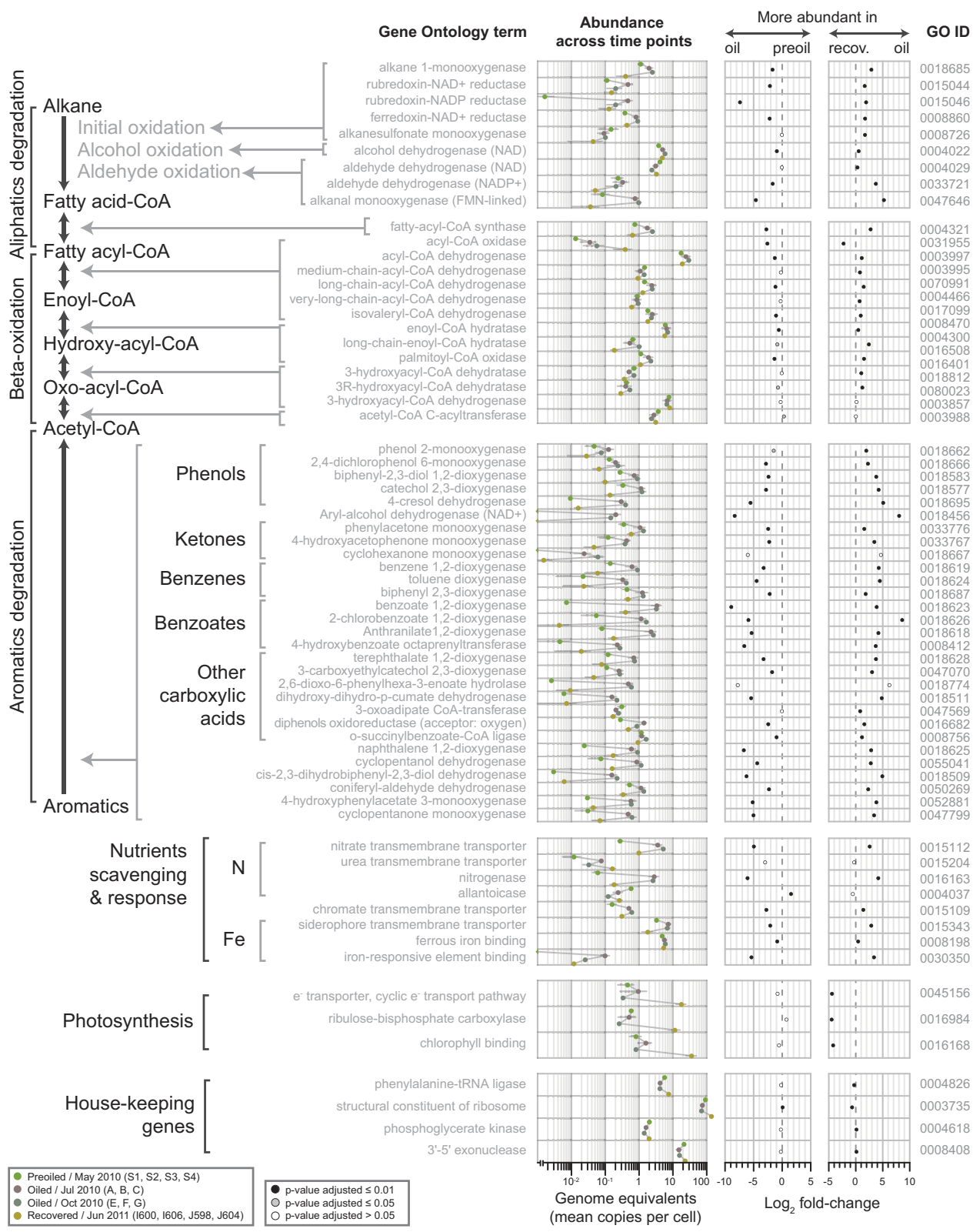

Figure 3 Microbial community functional shifts in response to oil. Selected molecular functions related to hydrocarbon degradation, nutrient scavenging and response, photosynthesis, and some house-keeping genes are listed (left) along with the mean genome equivalents per group of samples (middle) and the $\log _{2}$ of Preoil/Oiled and Oiled/Recovered fold changes (right). The rightmost column indicates the GO ID of the terms. The abundance was assessed as average genome equivalents (mean copies per bacterial/archaeal cell) on each sampling time (downwards; see legend). The triangles indicate values below the plotted range. The $\log _{2}$-fold-change was estimated as the $\log _{2}$ of the ratio of normalized counts between pre-oiled samples (S1, S2, S3, S4) and oiled samples (A, B, C, E, F, G); and between oiled samples and recovered samples (I600, I606, J598, J604). P-values were estimated using a negative binomial test.

Figure 4 Phylogenetic reconstruction of AlkB protein sequences and putative sequences recovered from the metagenomes. The tree displays reference AlkB (alkane hydrolase) proteins (text colored by clusters, following the nomenclature of Wang et al., 2010) along with variants assembled from the metagenomes (black text). Proteins with experimental evidence of activity (from heterologous expression or gene knockouts) are indicated by + . Reads mapping to different nodes of the tree are displayed as pie charts. The radius of the pie charts indicates the fraction of the metagenomes mapping to the node (expressed as reads per million, Reads mapping legend), and the different colors of the slices indicate the dataset of origin (Dataset legend). The terminal branch of the sequence A-87946 (cluster V) was shortened by 7.0 units, as indicated by a discontinuity. The right panel indicates the total abundance of each cluster averaged per group of datasets (in reads per million): Pre-oil samples (green), Oiled samples from July 2010 (mauve), Oiled samples from October 2010 (sea green) and recovered samples (olive). Reference sequences (including out-group sequence XylM from Pseudomonas putida) and clusters nomenclature (in squared parenthesis) are based on Wang et al. (2010), but the definition of the clusters (colored backgrounds) was broaden to include all sequences in the analysis, and two additional clusters were defined ('OS-I' and 'OS-II'). 
and siderophores (iron chelation) were observed to be increased in oiled samples, whereas functions related to primary production such as iron-responsive elements (iron-responsive binding), as well as functions related to photosynthesis (possibly transported from neighboring marine communities), were enriched in the recovered communities. Notably, most functional categories exhibiting statistically significant

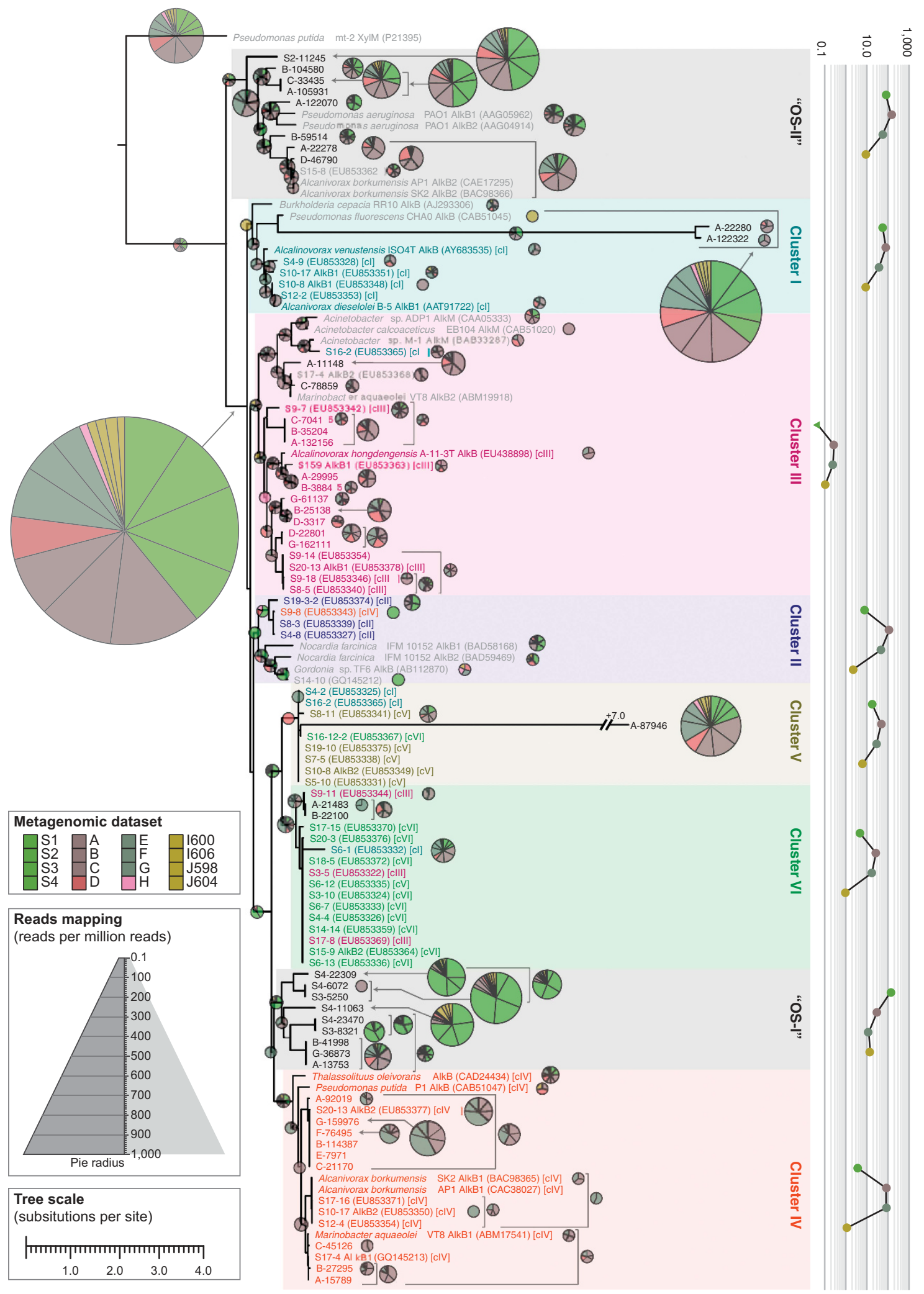


difference in abundance in the oiled communities returned to the pre-oil state in the recovered communities, in some cases exceeding their pre-oil levels (Figure 3 and Supplementary File S4).

To explore the phylogenetic diversity of genes related to oil degradation, we selected AlkB (alkane hydrolase) as a marker for alkane degradation and reconstructed a high-quality gene phylogeny based on 66 reference genes (mostly based on Wang et al., 2010) and 43 genes recovered from metagenomic assemblies. In addition, individual metagenomic reads from all datasets were assigned to the most likely node in the tree to provide a quantitative picture of the shifts of AlkB variants over time (Figure 4). This dataset included sequences from 14 different genera in five different classes, hence spanning a large diversity of known alkane degraders. Additionally, this dataset covered the diversity of the partial AlkB sequences reported by Smith et al. (2013) for the northern Gulf of Mexico, most of which were assigned to clusters IV (73.5\%) and II $(20.9 \%)$. As expected, very few reads from recovered samples were placed in the tree, and most placed reads were derived from oiled or weathered oil samples. However, an intermediate abundance was detected in pre-oil samples (Figure 3, first row: alkane 1-monooxygenase). In fact, only cluster III was undetectable in pre-oil samples, whereas all other clusters followed the general trend observed for the entire gene abundance ( $c f$. right panel on Figure 4 and first row on Figure 3). More importantly, the reads from different oiled and pre-oil samples were distributed across different clades, with larger concentrations in a few clades spanning the entire tree, that is, an uneven but phylogenetically unconstrained distribution. Notably, we identified a cluster formed exclusively by genes from this study (labeled 'OS-I' in Figure 4) most abundant in the pre-oil samples and negatively impacted by the oil spill.

\section{Population successional patterns and community recovery}

In addition to the large differences observed in community composition (both taxonomic and functional) between pre-oil, oiled and recovered samples, the microbial communities characterized in July 2010 differed from those in October 2010 (Figures $1 \mathrm{~b}$ and $\mathrm{c}$ ), concurrent with a significant reduction in total petroleum hydrocarbons (Figure 1a). Examination of the taxonomic distribution revealed that some populations responded rapidly, reaching high abundances in July 2010, with large reductions in abundance by October, and being barely detectable in the recovered samples of June 2011 (Figure 3c). These populations included members of the Alcanivorax, Borrelia, Spirochaeta, Micavibrio and Bacteroides genera. However, some populations were observed to peak in abundance in the October 2010 samples and significantly drop in the recovered samples, such as Hyphomonas,
Treponema, Sphingopyxis and Hirschia. Most oilassociated genera did not maintain their abundance in oiled samples of July and October 2010 with the notable exceptions of Marinobacter and Parvibaculum. The abundance profiles probably reflected organisms with different metabolic properties with respect to oil degradation such as fast responders to easily degradable oil constituents, organisms specialized in degradation of aromatic and more recalcitrant oil fractions, and oil degradation generalists. Finally, we identified a significant increase in minimum doubling time between oiled samples of July and October 2010 based on codon usage bias patterns (Vieira-Silva and Rocha, 2010) (difference of means: $3 \mathrm{~h} 9 \mathrm{~m}, P$-value $<10^{-16}$, two-sided $t$-test; Supplementary Figure S4A). The increase in doubling time observed from October 2010 to the recovered samples (June 2011) was much smaller and not statistically significant (difference of means: $27 \mathrm{~m}, P$-value: 0.19 , two-sided $t$-test). Altogether, these results indicate that the community response to the oil spill involved well-defined successional trends: a rapid response (from May to July 2010), with a peak growth rate in July 2010, followed by a continued decrease in taxonomic diversity (between May and October 2010) and, finally, a reduction in abundance of several known and suspected oil degraders, concomitant with the increase in abundance of several typical marine groups undetectable or rare in oiled samples, a large increase in taxonomic diversity and a decrease in functional diversity.

\section{Discussion}

The sands of the Pensacola Municipal Beach received repeated pulses of oil deposition for over a month, and, after about a year, oil was still detected in the beach sands, although it had concentrations below $5 \mathrm{mg} \mathrm{kg}^{-1}$ (Figure 1a). This indicates that the microbial community faced largely a press (long-term) disturbance given the time scale of microbial generation cycles and migration processes (Shade et al., 2012). Press disturbances often result in community shifts driven by the response traits of individual populations to the disturbance, presumably sensitivity to toxic compounds and hydrocarbon degradation capabilities in the case of oil contamination. The diversity and abundance of indigenous alkane-degraders preceding the oil spill in the beach ecosystem, as well as the origin of the degraders observed after the spill, was not robustly assessed in previous studies mostly owing to the incomplete diversity recovered in cultures of alkane-degraders and lack of complete understanding of their ecophysiology. The observation of a large and phylogenetically unconstrained diversity of alkB genes in the oiled and pre-oil samples supports the hypothesis that the response to the oil spill was primarily caused by shifts in abundances of pre-existing populations, as previously 
observed in the deep-sea oil plume (Hazen et al., 2010). In other words, the $a l k B$ genes present in the oiled communities were not derived from a single or a few recent gene alleles but, instead, a large diversity of degraders was latent in the sand and/or surface waters seeping into the sands before the oil spill.

Initial responders (July 2010) included members of the genera Alcanivorax, Borrelia, Spirochaeta, Micavibrio and Bacteroides, all members of the abundant fraction $(\geqslant 1 \%$ of the total community) in the oiled samples. Alcanivorax is a genus known for its hydrocarbonoclastic capabilities that can utilize alkanes but not aromatic hydrocarbons (Schneiker et al., 2006); the metabolic capabilities of the other genera in oil hydrocarbon degradation remain speculative. Interestingly, we found putative alkB genes (alkane hydrolase) in contigs classified as Alcanivorax, Borrelia and Bacteroides, but no evidence of arh $A$ (polycyclic aromatic hydrocarbon dioxygenase) in any of these genera, and putative nahA genes (naphthalene 1,2-dioxygenase) only in Alcanivorax. The former populations were replaced in the abundant fraction in October 2010 by members of the genus Treponema and the class Alphaproteobacteria (including Hyphomonas, Sphingopyxis and Hirschia), suggesting a successional dynamic as previously observed based on 16S rRNA gene amplicon data (Kostka et al., 2011; Lamendella et al., 2014). Members of the Hyphomonas genus have been reported as abundant members in consortia degrading aromatic compounds, which are typically more recalcitrant components of the crude oil than alkanes and hence, more prevalent in later post-spill stages (Maeda et al., 2009, 2010). Similarly, Sphingopyxis is known to have aromatic hydrocarbon degradation capabilities (Kertesz and Kawasaki, 2010) and was previously detected as a dominant group in soil-derived oil-degrading consortia amended with natural organic matter (Hassan et al., 2011).

Very few microbial groups, including members of the genera Marinobacter and Parvibaculum, were consistently enriched in the oiled samples with no noticeable change in abundance between July and October 2010. Putative alkB and cyp153 (cytochrome P450 family) genes, associated with alkane degradation, were identified in assembled contigs assigned to both genera, and putative nah $A$ genes, associated with aromatic hydrocarbon degradation, were identified in contigs classified as Marinobacter. Members of the Marinobacter genus are able to degrade a large variety of aliphatic and aromatic hydrocarbons (Gauthier et al., 1992). Similarly, members of the Parvibaculum genus exhibit metabolic capabilities for both aliphatic and aromatic degradation (Schneiker et al., 2006; Wang et al., 2010; Lai et al., 2011). In contrast to previous analyses based on 18S rRNA gene amplicons (Bik et al., 2012), no consistent, statistically significant shifts in the taxonomic composition of the eukaryotic fraction were detected between sampling dates or degree of oiling (Supplementary File S3).
Finally, in June 2011, Synechococcus, Pediococcus and archaeal genera including Nitrosopumilus, Cenarchaeum and Nitrosoarchaeum dominated the abundant fraction (in contrast to oiled samples), and an overall increase in the eukaryotic fraction was observed. Many of the former microbial groups are abundant in oligotrophic or nutrient-poor marine ecosystems, indicating that they represent the sensitive fraction of the community to the oil spill, but to a large extent the community was resilient, as generally observed in microbial communities (Allison and Martiny, 2008). Notably, the observed succession process exhibited signs of community recovery, but the community in June 2011, 1 year after the oil reached the shoreline, significantly differed from that in May 2010, before oiling, similar to the results of previous microcosm experiments on oil amendment of beach sediment inocula (Röling et al., 2002). The differences between the recovered community and its counterpart before the oil spill may be due to the long-term effects of the oil disturbance (for example, establishment of new taxa), stochastic events or other environmental factors such as organic matter input, nutrient input and salinity changes. Clearly, more samples and analyses would be required to obtain further insights into the latter issue. Nonetheless, our results also suggest that these sensitive marine groups could serve as indicator species of oil presence and toxicity in future oil spill studies, and thus, potentially provide useful information for guiding bioremediation efforts and decisions by site managers.

In general, microbial communities changed both taxonomically and functionally after exposure to a range of petroleum hydrocarbon concentrations. The community shifts caused a decrease in taxonomic diversity during May to October 2010, with a significant recovery by June 2011 (Supplementary Figure S3C). Interestingly, the functional diversity was observed to follow a contrasting trend: it increased between May and July 2010, was maintained between July and October 2010, and significantly decreased in June 2011 (Supplementary Figure S3A-B). We hypothesize that several oligotrophic (specialized) taxa were strongly outcompeted upon deposition of oil onshore. Growth arrest due to limited hydrocarbon degradation capabilities and/or sensitivity to toxic compounds from the continued presence of oil onshore would impact more severely oligotrophic and/or specialist than copiotrophic and/or generalist populations. Hence, a significant reduction in taxonomic diversity but not functional diversity was expected, as observed in these communities. Moreover, we provided evidence indicating that specific fast-growing organisms (typically assumed to be copiotrophic) thrived in the presence of relatively high concentrations of petroleum hydrocarbons. In other words, the disturbance favored generalist organisms in the communities, and the post-disturbance communities 
were characterized by a narrower set of more specialized functions. This observation seems counterintuitive because a common expectation is that a press disturbance would exclusively select for few highly specialized organisms, in this case oildegraders. Nevertheless, this trend is predicted by the disturbance-specialization hypothesis (Vázquez and Simberloff, 2002), is consistent with ecological succession theory and was previously observed in plant communities (for example, Bazzaz and Pickett, 1980). It should be mentioned, however, that the patterns observed here might be specific to disturbances from crude oil and sand beach ecosystems and not immediately generalizable to other, more selective disturbances (Röling and van Bodegom, 2014). Crude oil is composed of tens of thousands of different carbon sources that would favor generalists in early succession, as well as toxic compounds that would preferentially select against specialists. Sand beaches in the Gulf of Mexico are characterized by low carbon content and nutrient-poor conditions relative to marshes or other coastal sediments (Huettel et al., 2014). This could explain the relative abundance of putative chemolithoautotrophic archaea (Nitrosopumilus, Cenarchaeum) in the recovered communities and suggests a suppression of a range of organisms that are adapted to carbonlimited conditions.

In summary, the community response was primarily characterized by two concomitant trends. First, most of the community is selected based on the ability to survive under disturbed conditions, that is, the response to the disturbance correlates negatively with the level of specialization. Second, few organisms with traits selected by the disturbance become highly abundant, as niche opportunity promotes invasion (Shea and Chesson, 2002; Pintor et al., 2011). Overall, our results provide evidence of complex successional patterns in the studied communities, involving invasion promoted by capabilities for oil hydrocarbon degradation, as well as population survival generally hindered by specialization and susceptibility to oil toxicity, and a general recovery of diversity, specialization and sensitive marine groups a year after the disturbance.

\section{Conflict of Interest}

The authors declare no conflicts of interest.

\section{Acknowledgements}

This work was supported in part by the U.S. National Science Foundation (NSF) award no. 1241046 (to KTK), OCE-1057417 and OCE-1044939 (to MH, JEK), the NSF graduate research fellowship no. 2013172310 (to WAO), and by a grant from BP/The Gulf of Mexico Research Initiative to the Deep-C Consortium (\#SA 12-12, GoMRI-008). We thank Patrick Chain and the personnel of the Los Alamos National Laboratory for sequencing of the samples.

\section{References}

Allison SD, Martiny JBH. (2008). Resistance, resilience, and redundancy in microbial communities. Proc Natl Acad Sci USA 105: 11512-11519.

Altschul SF, Gish W, Miller W, Myers EW, Lipman DJ. (1990). Basic local alignment search tool. J Mol Biol 215: 403-410.

Anders S, Huber W. (2010). Differential expression analysis for sequence count data. Genome Biol 11: R106.

Atlas RM, Hazen TC. (2011). Oil biodegradation and bioremediation: a tale of the two worst spills in U.S. history. Environ Sci Technol 45: 6709-6715.

Bazzaz FA, Pickett STA. (1980). Physiological ecology of tropical succession: a comparative review. Annu Rev Ecol Syst 11: 287-310.

Bengtsson J, Eriksson KM, Hartmann M, Wang Z, Shenoy BD, Grelet G-A et al. (2011). Metaxa: a software tool for automated detection and discrimination among ribosomal small subunit (12 S/16 S/18 S) sequences of archaea, bacteria, eukaryotes, mitochondria, and chloroplasts in metagenomes and environmental sequencing datasets. Antonie Van Leeuwenhoek 100: $471-475$.

Berger SA, Krompass D, Stamatakis A. (2011). Performance, accuracy, and web server for evolutionary placement of short sequence reads under maximum likelihood. Syst Biol 60: 291-302.

Berthe-Corti L, Nachtkamp M. (2010). Bacterial communities in hydrocarbon-contaminated marine coastal environments. In: Timmis KN (ed), Handbook of Hydrocarbon and Lipid Microbiology. Springer: Berlin Heidelberg, pp 2349-2359.

Bik HM, Halanych KM, Sharma J, Thomas WK. (2012). Dramatic shifts in benthic microbial eukaryote communities following the Deepwater Horizon oil spill. PLoS One 7: e38550.

Bokulich NA, Subramanian S, Faith JJ, Gevers D, Gordon JI, Knight R et al. (2013). Quality-filtering vastly improves diversity estimates from Illumina amplicon sequencing. Nat Methods 10: 57-59.

Chao A. (1984). Nonparametric estimation of the number of classes in a population. Scand J Stat 11: 265-270.

Chao A, Shen T-J. (2003). Nonparametric estimation of Shannon's index of diversity when there are unseen species in sample. Environ Ecol Stat 10: 429-443.

Dethlefsen L, Schmidt TM. (2007). Performance of the translational apparatus varies with the ecological strategies of bacteria. J Bacteriol 189: 3237-3245.

Dupont CL, Rusch DB, Yooseph S, Lombardo M-J, Richter RA, Valas $\mathrm{R}$ et al. (2012). Genomic insights to SAR86, an abundant and uncultivated marine bacterial lineage. ISME J 6: 1186-1199.

Edgar RC. (2004). MUSCLE: multiple sequence alignment with high accuracy and high throughput. Nucleic Acids Res 32: 1792-1797.

Edgar RC. (2010). Search and clustering orders of magnitude faster than BLAST. Bioinformatics 26: 2460-2461.

Gauthier MJ, Lafay B, Christen R, Fernandez L, Acquaviva M, Bonin P et al. (1992). Marinobacter hydrocarbonoclasticus gen. nov., sp. nov., a new, extremely halotolerant, hydrocarbon-degrading marine bacterium. Int J Syst Bacteriol 42: 568-576.

Greer CW. (2010). Bacterial diversity in hydrocarbonpolluted rivers, estuaries and sediments. In: Timmis KN (ed), Handbook of Hydrocarbon and Lipid Microbiology. Springer: Berlin Heidelberg, pp 2329-2338. 
Hassan A, Taqi Z, Obuekwe C, Al-Saleh E. (2011). Characterization of crude oil-degrading bacteria in a crude oil-contaminated and uncontaminated site in Kuwait. WIT Trans Ecol Envir 167: 177-186.

Hausser J, Strimer K. (2013). Entropy: estimation of entropy, mutual information and related quantities. http://cran.r-project.org/web/packages/entropy/index. html. Accessed August 16, 2014.

Hayworth JS, Clement TP, Valentine JF. (2011). Deepwater Horizon oil spill impacts on Alabama beaches. Hydrol Earth Syst Sci 15: 3639-3649.

Hazen TC, Dubinsky EA, DeSantis TZ, Andersen GL, Piceno YM, Singh N et al. (2010). Deep-sea oil plume enriches indigenous oil-degrading bacteria. Science 330: 204-208.

Head IM, Jones DM, Röling WFM. (2006). Marine microorganisms make a meal of oil. Nat Rev Microbiol 4: 173-182.

Huettel M, Berg P, Kostka JE. (2014). Benthic exchange and biogeochemical cycling in permeable sediments. Annu Rev Mar Sci 6: 23-51.

Joye SB, Teske AP, Kostka JE. (2014). Microbial dynamics following the macondo oil well blowout across Gulf of Mexico environments. BioScience 64: 766-777.

Kent WJ. (2002). BLAT-the BLAST-like alignment tool. Genome Res 12: 656-664.

Kertesz MA, Kawasaki A. (2010). Hydrocarbon-degrading Sphingomonads: Sphingomonas, Sphingobium, Novosphingobium, and Sphingopyxis. In: Timmis KN (ed), Handbook of Hydrocarbon and Lipid Microbiology. Springer: Berlin Heidelberg, pp 1693-1705.

King GM, Kostka JE, Hazen TC, Sobecky PA. (2015). Microbial responses to the Deepwater Horizon oil spill in the northern Gulf of Mexico: from coastal wetlands to the deep sea. Annu Rev Mar Sci 7: 377-401.

Konstantinidis KT, Tiedje JM. (2005). Genomic insights that advance the species definition for prokaryotes. Proc Natl Acad Sci USA 102: 2567-2572.

Kostka JE, Prakash O, Overholt WA, Green SJ, Freyer G, Canion A et al. (2011). Hydrocarbondegrading bacteria and the bacterial community response in Gulf of Mexico beach sands impacted by the Deepwater Horizon oil spill. Appl Environ Microbiol 77: 7962-7974.

Kostka JE, Teske A, Joye SB, Head IM. (2014). The metabolic pathways and environmental controls of hydrocarbon biodegradation in marine ecosystems. Front Microbiol 5: 471.

Lai Q, Wang L, Liu Y, Yuan J, Sun F, Shao Z. (2011). Parvibaculum indicum sp. nov., isolated from deep-sea water. Int J Syst Evol Microbiol 61: 271-274.

Lamendella R, Strutt S, Borglin SE, Chakraborty R, Tas N, Mason OU et al. (2014). Assessment of the Deepwater Horizon oil spill impact on Gulf coast microbial communities. Aquat Microbiol 5: 130.

Lubchenco J, McNutt M, Lehr B, Sogge M, Miller M, Hammond S et al. (2010). Deepwater Horizon/BP Oil Budget: What happened to the oil? http://www.noaanews. noaa.gov/stories2010/PDFs/OilBudget_description_\% 2083final.pdf.

Luo C, Rodriguez-R LM, Konstantinidis KT. (2014). MyTaxa: an advanced taxonomic classifier for genomic and metagenomic sequences. Nucleic Acids Res 42: e73-e73.

Lu Z, Deng Y, Van Nostrand JD, He Z, Voordeckers J, Zhou A et al. (2012). Microbial gene functions enriched in the Deepwater Horizon deep-sea oil plume. ISME J 6: $451-460$.
Maeda R, Ito Y, Iwata K, Omori T. (2010). Comparison of marine and terrestrial carbazole-degrading bacteria. Curr Res Technol Educ Top Appl Microbiol Microb Biotechnol 2: 1311-1321.

Maeda R, Nagashima H, Widada J, Iwata K, Omori T. (2009). Novel marine carbazole-degrading bacteria. FEMS Microbiol Lett 292: 203-209.

Matsen FA, Kodner RB, Armbrust EV. (2010). pplacer: linear time maximum-likelihood and Bayesian phylogenetic placement of sequences onto a fixed reference tree. BMC Bioinformatics 11: 538

McGenity TJ, Folwell BD, McKew BA, Sanni GO. (2012). Marine crude-oil biodegradation: a central role for interspecies interactions. Aquat Biosyst 8: 10.

Michel J, Owens EH, Zengel S, Graham A, Nixon Z, Allard T et al. (2013). Extent and degree of shoreline oiling: Deepwater Horizon Oil Spill, Gulf of Mexico, USA Chin, W-C (ed). PLoS One 8: e65087.

Newton RJ, Huse SM, Morrison HG, Peake CS, Sogin ML, McLellan SL. (2013). Shifts in the microbial community composition of Gulf Coast beaches following beach oiling. PLoS One 8: e74265.

Pintor LM, Brown JS, Vincent TL. (2011). Evolutionary game theory as a framework for studying biological invasions. Am Nat 177: 410-423.

Poretsky R, Rodriguez-R LM, Luo C, Tsementzi D, Konstantinidis KT. (2014). Strengths and limitations of $16 \mathrm{~S}$ rRNA gene amplicon sequencing in revealing temporal microbial community dynamics. PLoS One 9: e93827.

Prosser JI, Bohannan BJM, Curtis TP, Ellis RJ, Firestone MK, Freckleton RP et al. (2007). The role of ecological theory in microbial ecology. Nat Rev Microbiol 5: 384-392.

Reed HE, Martiny JBH. (2007). Testing the functional significance of microbial composition in natural communities. FEMS Microbiol Ecol 62: 161-170.

Rho M, Tang H, Ye Y. (2010). FragGeneScan: predicting genes in short and error-prone reads. Nucleic Acids Res 38: e191.

Rodriguez-R LM, Konstantinidis KT. (2014a). Estimating coverage in metagenomic data sets and why it matters. ISME J 8: 2349-2351.

Rodriguez-R LM, Konstantinidis KT. (2014b). Nonpareil: a redundancy-based approach to assess the level of coverage in metagenomic datasets. Bioinformatics 30: 629-635.

Röling WFM, van Bodegom PM. (2014). Toward quantitative understanding on microbial community structure and functioning: a modeling-centered approach using degradation of marine oil spills as example. Aquat Microbiol 5: 125.

Röling WFM, Milner MG, Jones DM, Fratepietro F, Swannell RPJ, Daniel F et al. (2004). Bacterial community dynamics and hydrocarbon degradation during a field-scale evaluation of bioremediation on a mudflat beach contaminated with buried oil. Appl Environ Microbiol 70: 2603-2613.

Röling WFM, Milner MG, Jones DM, Lee K, Daniel F, Swannell RJP et al. (2002). Robust hydrocarbon degradation and dynamics of bacterial communities during nutrient-enhanced oil spill bioremediation. Appl Environ Microbiol 68: 5537-5548.

Schneiker S, dos Santos VAM, Bartels D, Bekel T, Brecht M, Buhrmester J et al. (2006). Genome sequence of the ubiquitous hydrocarbon-degrading marine bacterium Alcanivorax borkumensis. Nat Biotechnol 24: 997-1004. 
Shade A, Peter H, Allison SD, Baho DL, Berga M, Bürgmann $\mathrm{H}$ et al. (2012). Fundamentals of microbial community resistance and resilience. Front Microbiol 3: 417.

Shea K, Chesson P. (2002). Community ecology theory as a framework for biological invasions. Trends Ecol Evol 17: 170-176.

Sievers F, Wilm A, Dineen D, Gibson TJ, Karplus K, Li W et al. (2011). Fast, scalable generation of high-quality protein multiple sequence alignments using Clustal Omega. Mol Syst Biol 7: 539.

Smith CB, Tolar BB, Hollibaugh JT, King GM. (2013). Alkane hydroxylase gene (alkB) phylotype composition and diversity in northern Gulf of Mexico bacterioplankton. Aquat Microbiol 4: 370.

Stamatakis A. (2006). RAxML-VI-HPC: maximum likelihood-based phylogenetic analyses with thousands of taxa and mixed models. Bioinformatics 22: 2688-2690.

Suzek BE, Huang H, McGarvey P, Mazumder R, Wu CH. (2007). UniRef: comprehensive and non-redundant UniProt reference clusters. Bioinformatics 23: 1282-1288.

Valentine DL, Mezić I, Maćešić S, Črnjarić-Žic N, Ivić S, Hogan PJ et al. (2012). Dynamic autoinoculation and the microbial ecology of a deep water hydrocarbon irruption. Proc Natl Acad Sci USA 109: 20286-20291.
Vázquez DP, Simberloff D. (2002). Ecological specialization and susceptibility to disturbance: conjectures and refutations. Am Nat 159: 606-623.

Vieira-Silva S, Rocha EPC. (2010). The systemic imprint of growth and its uses in ecological (meta)genomics. PLoS Genet 6: e1000808.

Wang L, Wang W, Lai Q, Shao Z. (2010). Gene diversity of CYP153A and AlkB alkane hydroxylases in oil-degrading bacteria isolated from the Atlantic Ocean. Environ Microbiol 12: 1230-1242.

Wang P, Roberts TM. (2013). Distribution of surficial and buried oil contaminants across sandy beaches along NW Florida and Alabama coasts following the Deepwater Horizon oil spill in 2010. J Coast Res 291: 144-155.

Wang Q, Garrity GM, Tiedje JM, Cole JR. (2007). Naïve bayesian classifier for rapid assignment of rRNA sequences into the new bacterial taxonomy. Appl Environ Microbiol 73: 5261-5267.

Yakimov MM, Timmis KN, Golyshin PN. (2007). Obligate oil-degrading marine bacteria. Curr Opin Biotechnol 18: $257-266$.

Zuijdgeest A, Huettel M. (2012). Dispersants as used in response to the MC252-spill lead to higher mobility of polycyclic aromatic hydrocarbons in oil-contaminated Gulf of Mexico sand. PLoS One 7: e50549.

Supplementary Information accompanies this paper on The ISME Journal website (http://www.nature.com/ismej) 